\title{
Superior vena cava syndrome caused by a pseudoaneurysm of the ascending aorta
}

\author{
T Vydt, J Coddens, F Wellens
}

Heart 2005;91:e29 (http://www.heartjnl.com/cgi/content/full/91/4/e29). doi: 10.1136/hrt.2004.053355

Pseudoaneurysm of the ascending aorta is a well known complication after aortic root surgery. A case of a large pseudoaneurysm is reported, seen as a superior vena cava syndrome, a very rare clinical presentation. Perioperative transoesophageal echocardiography showed the presence of a large pseudoaneurysm starting from the left coronary ostium implantation.

A 56 year old man was seen at our emergency department complaining of exertion dyspnoea lasting two days. He had undergone an elective replacement of the aortic valve because of severe aortic regurgitation 14 years earlier with a Björk-Shiley $29 \mathrm{~mm}$ Monostrut mechanical valve. Six years later he presented with a rupture of an ascending aortic aneurysm with a maximum diameter of $10 \mathrm{~cm}$. This was urgently treated with a Bentall reconstruction (inclusion and wrap technique) with a St Jude Medical $25 \mathrm{~mm}$ composite graft. Warfarin was his only medication.

On admission, the patient was afebrile, with a blood pressure of 132/76 $\mathrm{mm} \mathrm{Hg}$, and his oxygen saturation without supplemental oxygen was $85 \%$. Cardiac examination found an irregular rhythm at 143 beats/min and normal heart sounds consistent with a mechanical prosthetic valve. The jugular veins were distended bilaterally to the angle of the mandible. Cyanosis and swelling of the head and neck were noticed. Lungs were clear. Superior vena cava syndrome (SVCS) was clinically diagnosed. The remainder of his physical examination was normal.

The ECG showed atrial fibrillation with fast ventricular response ( 143 beats/min). Chest radiography was remarkable for widening of the mediastinum compatible with dilatation of the ascending aorta. Laboratory data included a prothrombin time of $15 \%$. Cardiac enzymes were negative. Bedside transthoracic echocardiography showed normal left ventricular contractility, a normal functioning aortic prosthetic valve, and a large pseudoaneurysm of the ascending aorta. Urgent computed tomography of the chest showed a pseudoaneurysm of the ascending aorta with a maximum diameter of $10 \mathrm{~cm}$ and an important amount of thrombotic material, and a secondary SVCS with extensive collateral circulation through the vena azygos system (fig 1).

A pulsatile flow was felt in the region of the vena femoralis, which suggested a fistulisation of the pseudoaneurysm to the right atrium. Urgent femoro-femoral cannulation was performed, extracorporeal circulation was started, and the patient's temperature was lowered to $20^{\circ} \mathrm{C}$. Perioperative transoesophageal echocardiography showed that the pseudoaneurysm originated in the left coronary ostium anastomosis (fig 2). After repeat sternotomy the ascending aorta was cross clamped and the caval veins were snared. Retrograde cardioplegia was installed. The large pseudoaneurysm was incised, with complete dehiscence from the left coronary ostium and the prosthesis leaving a gap of $2 \mathrm{~cm}$ between the structures. The pseudoaneurysm was also perforating into the right atrium causing an important left to right shunt. This was closed with a bovine pericardial patch. There was also a minor leak originating from the right coronary ostium anastomosis, which was sutured. A Dacron graft was used to reanastomose the left coronary artery. The aortic valve prosthesis and the aortic graft were considered to be functional and were left in situ. The native aortic wall was partially resected and not wrapped around the graft.

The postoperative course was uneventful. Ten days after admission the patient was discharged in good condition. One year after discharge, the patient was still asymptomatic. Clinical, echocardiographic, and radiographic findings were all within normal range.

\section{DISCUSSION}

A pseudoaneurysm of the ascending aorta is a well known complication after cardiovascular surgery. Nonetheless, it still is a rare clinical entity but potentially fatal if left untreated.

The potential sites for pseudoaneurysm formation after cardiac surgery are the sites of aortic cross clamp, aortotomy,

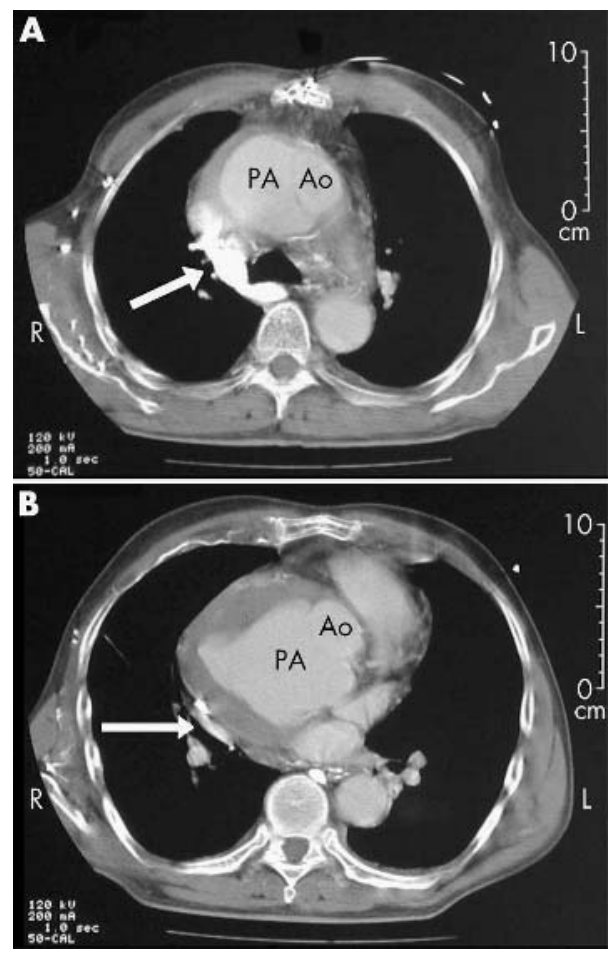

Figure 1 Contrast enhanced computed tomography of the superior mediastinum showing (panel A) a dilated azygos system (arrow), the pseudoaneurysm (PA) of the aorta (Ao) and (panel B) compression of the superior vena cava (arrow). 


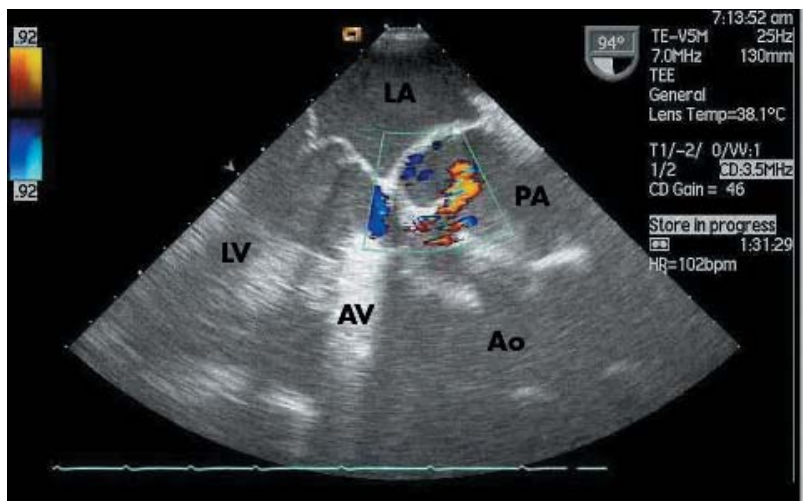

Figure 2 Perioperative transoesophageal echocardiography showing the origin of the PA and dehiscence of the left coronary artery with a jet (colour Doppler) from the anastomosis of the left coronary artery to the space between the aortic prosthesis and the native aorta. AV, aortic valve; LA, left atrium; LV, left ventricle.

proximal or distal aortic suture lines, aortic cannulation, cardioplegia needle, aortic vent, and coronary anastomosis. ${ }^{1}$ The mechanism of pseudoaneurysm formation is not completely understood, but suture line tension and persistent bleeding into the space between the graft and the wrapped aorta wall seem to be most important. ${ }^{3}$

SVCS is a clinical entity resulting from intravascular obstruction or extrinsic compression. McIntire and Sykes ${ }^{4}$ reported in 1949 a series of 502 SVCS cases. Of these, 67\% were due to benign conditions, of which $30 \%$ were secondary to aortic (syphilitic) aneurysms and 15\% secondary to chronic mediastinitis, whereas $33 \%$ were due to malignancy. In the past decades, the common causes of SVCS have changed importantly. In recent reports, benign diseases accounted for only $10 \%$ of cases. ${ }^{5}$ Nowadays SVCS is almost solely seen as a presentation caused by malignancy. At presentation benign causes of SVCS are too often overlooked in the differential diagnosis. Besides benign intrathoracic tumours and inflammatory or infective processes, a group of cardiovascular diseases can cause a SVCS. Dehiscence of a coronary artery producing pseudoaneurysm formation is a very rare cause of SVCS.

Bentall and DeBono ${ }^{6}$ introduced in 1968 a technique for replacing the ascending aorta and aortic valve with a composite graft containing a prosthetic valve with end to side reimplantation of the coronary artery ostia, which resulted in a significantly prolonged life expectancy of patients with combined disorders of the ascending aorta and aortic valve. ${ }^{4}$ Some modifications have since been made.

Despite the lack of prospective randomised studies, standard use of the open and button technique (instead of the original inclusion and wrap technique), together with an increasing operative experience, the evolution of pump oxygenator systems and graft materials, and a smaller threshold to quick re-exploration, has resulted in a smaller need for wrapping the aorta tightly around the graft, thereby reducing the tension on the suture lines and so the risk for pseudoaneurysm formation. ${ }^{137}$ In most series the incidence of pseudoaneurysms with the inclusion technique ranged from 7-25\%. ${ }^{1}$ Kouchoukos et $a l^{1}$ and others ${ }^{7}$ observed an absence or a low incidence of pseudoaneurysm formation when the inclusion technique was not used.

Bleeding from the coronary ostial anastomosis can occur long after an aortic composite graft operation, especially when the inclusion and wrap technique has been used. We believe that our case is a dramatic example of this, with a massive pseudoaneurysm eight years postoperatively seen as an SVCS, a very rare clinical presentation.

\section{Authors' affiliations}

T Vydt, Department of Cardiology, AZ Middelheim, Antwerp, Belgium J Coddens, Department of Anaesthesiology, OLV-Hospital, Aalst,

Belgium

F Wellens, Department of Cardiothoracic Surgery, OLV-Hospital, Aalst, Belgium

Correspondence to: Dr Tom Vydt, Dr Van Raemdonckstraat 10, 9100 Sint-Niklaas, Belgium; Tom.Vydt@skynet.be

Accepted 1 November 2004

\section{REFERENCES}

1 Kouchoukos NT, Wareing TH, Murphy SF, et al. Sixteen-year experience with aortic root replacement: results of 172 operations. Ann Surg $1991 ; 214: 308-20$

2 Sullivan KL, Steiner RM, Smullens SN, et al. Pseudoaneurysm of the ascending aorta following cardiac surgery. Chest 1988;93:138-43.

3 Carrel T, Pasic M, Jenni R, et al. Reoperations after operation on the thoracic aorta: etiology, surgical techniques, and prevention. Ann Thorac Surg 1993;56:259-69.

4 Mclntire FT, Sykes EM. Obstruction of the superior vena cava: a review of the literature and report of two personal cases. Ann Intern Med 1949;30:925-60.

5 Baker GL, Barnes HJ. Superior vena cava syndrome: etiology, diagnosis and treatment. Am J Crit Care 1992;1:54-64.

6 Bentall H, DeBono A. A technique for complete replacement of the ascending aorta. Thorax 1968;23:338-9.

7 Kouchoukos NT, Marshall WG, Wedige-Stecher TA. Eleven-year experience with composite graft replacement of the ascending aorta and aortic valve. J Thorac Cardiovasc Surg 1986;92:691-705. 